\title{
Trunked Radio Solutions for Special Applications
}

\author{
Sławomir Gajewski, Małgorzata Gajewska, and Ryszard J. Katulski
}

\begin{abstract}
In the paper modern concepts of radio communication trunking-dispatch systems for special applications are presented. Basic standards of TETRA, DMR, and cdma2000 are mentioned. The aim of the paper is to present innovative trunking solutions based on the LTE system working both in FDD and TDD mode. The architecture of LTE trunked radio is shown as well as new services possible to implementation are described. The way of TETRA and LTE integration is characterized.
\end{abstract}

Keywords—LTE, trunked radio, TETRA, DMR

\section{INTRODUCTION}

$\mathbf{M}$ ILITARY and civil services and institutions responsible for national security and defence of borders seek system solutions for realization of dispatch services of high quality. Till now, dominant role play systems dedicated to these solutions as TETRA (Terrestrial Trunked Radio) and DMR (Digital Mobile Radio). There is no doubt that these systems meet the needs of customers, especially in the dispatching function, voice services, and transmission security. Therefore, they are still the main solutions used in practice.

However, it is impossible not to notice a very dynamic development of public cellular systems such as GSM, UMTS, and now - LTE (Long Term Evolution) that have changed and continue to change the face of modern radio communication. We know that the standardization of TETRA and DMR is still dynamic, and these systems are in fact safe tool for services. Both professional systems have many advantages which are very important from the point of view of the uniformed services, e.g. rail services, border guards, police, army etc. However, they put in return a number of limitations.

We see that the technological development of both TETRA and DMR, also due to narrow frequency channels available, not keeping pace with the modern system of public areas, which offer increasingly higher data rate and a very wide range of services.

In addition, TETRA and DMR require building an expensive infrastructure. So, it is a great financial problem of uniformed services. For this reason, the pace of development of these systems and their modernization is very slow and strongly limited, in particular, in terms of spectral efficiency and transmission rate.

All authors are with Gdansk University of Technology, Faculty of Electronics, Telecommunications and Informatics, Department of Radio Communication Systems and Networks, 11/12 Narutowicza Str., PL80233 Gdansk, Poland (e-mails: slagaj@eti.pg.gda.pl,m.gajewska@eti.pg.gda.pl, rjkat@eti.pg.gda.pl).
Despite the many advantages of TETRA and DMR as safe systems for the services with very limited access from the outside, many telecom operators for years see weakness of their deployment.

Therefore, in recent years, more time is spent using public cellular systems to the needs of specific applications. This carries a lot of restrictions but opens new service possibilities. These solutions allow separation of some system resources for the purpose of dispatching solutions. Widespread cellular systems typically offer a much higher frequency bandwidth, spectral efficiency, and achieved data rate as well as enable the use of ready-made infrastructure, which is their obvious advantage.

Everything seems to indicate that the solutions for the needs of uniformed services based on mainstream technologies can be dominant in the coming years. Particularly noteworthy are hybrid solutions that combine the TETRA functions with other mobile systems, especially LTE. At this moment of time, the integration should be expected primarily at the level of implementation of some services that require high bandwidth (e.g. video signal transmission, moving images, etc.). However, all the services and features of TETRA can remain at its level, maintaining the containment system and the high level of transmission security.

This approach can be justified, because it allows maintaining a significant independence of TETRA and its liberation from the need to implement broadband services, while extending the terminal functions, which in general are not adapted. Although under the TETRA standard currently developing TEDS technology (TETRA Enhanced Data Service) that allows data transmission at higher bit rates, but the services offered there will never be competition for LTE.

\section{BASIC STANDARDS}

As the main standard of trunking-dispatch system, especially in Europe, TETRA should be considered, which the dominant solution for the uniformed services is now. Similarly, in Poland dominate dispatching solutions based on TETRA technology.

Additionally, very popular in Poland is the DMR system, in fact, very similar to TETRA [1] but also possible to implement simplified and cheaper variants which for some customers are sufficient. There are also known simple dispatching solutions based on cdma2000 technology, popular in the United States but also implemented in Poland.

TETRA as the primary standard is an open standard digital radio communications for trunked-dispatch communication, based entirely on digital data transmission and mobile system concept [2, 3]. In general case, the transmission of speech and /or data is done through base stations. In the TETRA system, 
classic multiple access technology based on TDMA and FDMA is implemented.

It enables access to every radio channel with a bandwidth of $25 \mathrm{kHz}$, four time slots for the system users and / or to transmit control data. The quadrature, differential phase modulation $\pi / 4$ DQPSK is used.

Currently, in the system more flexible way to use TETRA radio channels is proposed and the improved performance of data services by standardizing TEDS subsystem. The subsystem that allowed a combination of several frequency channels in multiples of $25 \mathrm{kHz}$, reaching a width of 50, 100 or $150 \mathrm{kHz}$. This is done by using the basic $\pi / 8$ D8PSK modulation and QAM, implemented on multiple subcarriers (so-called Filtered Multi-Tone modulation - FMT). FMT is similar to OFDM (Orthogonal Frequency Division Multiplexing) but additional filtration of symbols transmitted in subchannels is made. This improves signals immunity to disturbance, especially those related to the Doppler effect, and reaches a slightly higher spectrum density. TEDS achieves data rates of several hundred kbps.

The main TETRA modes include working in the oldest variant, so-called V + D (Voice + Data), in which we have the possibility of simultaneous or independent transmission of both voice signals and data. There is also the PDO packetmode transmission (Packet Data Optimized) but it is not too popular.

In addition, TETRA terminals provides the opportunity to work in trunked mode (Trunking Mode Operation - TMO), using base stations, as well as direct-mode (Direct Mode Operation - DMO), which allows direct communication between terminals without a base station.

The DMR is standardized by ETSI, like TETRA, and is an alternative to TETRA, possible to implement in simplified variants a bit cheaper than TETRA (is more flexible from the point of view of its implementation). DMR is very popular in Poland because it offers greater possibility to reduce the required equipment in relation to TETRA. Therefore, it is believed that DMR is a less expensive in the costs of purchase and maintenance. DMR can operate in three different configurations of various complexities of hardware and services.

The first option is an unlicensed solution, designed for small business and private use. Allocated frequency band is unlicensed (approx. $446 \mathrm{MHz}$ ). Small powers of transmitted signals are applied and direct mode of communication between transceivers, without the mediation of base stations. This variant is dedicated mainly to the speech signal transmission services.

The second variant includes a conventional digital transmission. The system is more comprehensive and offers a rich set of services typical for dispatching systems, including data transmission. The solution is rather local but is most prevalent.

The third option is a typical trunked radio, equivalent to the TETRA system. In this embodiment, the system is ready to perform all trunking functions in WAN, using the network of base stations. Next to voice services, enables the transmission of packet-switched data.

In DMR the TDMA multiple access is implemented which enables access to two time slots in a single radio channel of $12.5 \mathrm{kHz}$ bandwidth and thus the two communication channels. 4FSK modulation is used and the detection and correction channel coding.

\section{GOTA SYSTEMS}

The next interesting solution is a set of systems called GoTa. The most famous solutions is the system based on cdma2000 technology working in $450 \mathrm{MHz}$ band (i.e. CDMA450) in Poland. The cdma2000 system is increasingly used in Poland by generalizing the cdma2000 cellular systems for internet applications. But the use of cdma2000 for dispatch solutions is popular, too.

As well known, the cdma2000 system is standardized by 3GPP2 as 3G system. This system is growing as fast as UMTS and is also based on the technique of direct sequence spread spectrum DS-CDMA. The cdma2000 is very popular in many countries in the world, especially in USA.

Dispatching functions have been designed in the system as a kind of overlay solution. The system is called a Digital Dispatch Communication System. This system has nearly 95\% coverage in Poland and enables the realization of basic dispatch services. But this is not so professional system like TETRA and DMR. Therefore, this solution is not recommended for use by the services, railways and border guards in Europe.

GoTa systems can be implemented in UMTS, and GSM, too, and these solutions are becoming increasingly popular in Europe.

However, the range of services offered in the cdma2000 system is very rich: broadcast connections, individual and group connections, alarm connections. Easy creation of user groups is possible and dispatch management. From this point of view, the system is similar to TETRA, and enables the broadband data services in a slightly greater extent.

The great drawback of the cdma2000system is a lack of feasibility of calls in direct transmission mode. This mode is admittedly through a complementary analogue transmission but this solution is rather primitive. From the other side, transmission security solutions are not compatible with European requirements, expected for uniformed services.

Now, GoTa 4G solutions are proposed, too. In general, this solution is based on LTE system working in the TDD mode. This shows that the importance of the development of LTE dispatch systems is increasing.

The main system component of GoTa $4 \mathrm{G}$ is so-called Commanding Dispatch System which can be connected to different subsystems and networks:

- Integrated system servers e.g.: recording server, contingency plan server, GIS (Global Information System) server, SMS server, fax server etc.

- Three screen dispatch console

- Wireless trunking systems: GoTa, TETRA, DMR, PDT (Police Digital Trunking system in China)

- Shortwave and ultrashort wave subsystems (radio stations)

- Video conference and video surveillance equipment

- Satellite communication system

- Conference room equipment: video conference, sound console, conference terminals

- Internal network equipment: analog phones, video phones, Ip phones etc. 


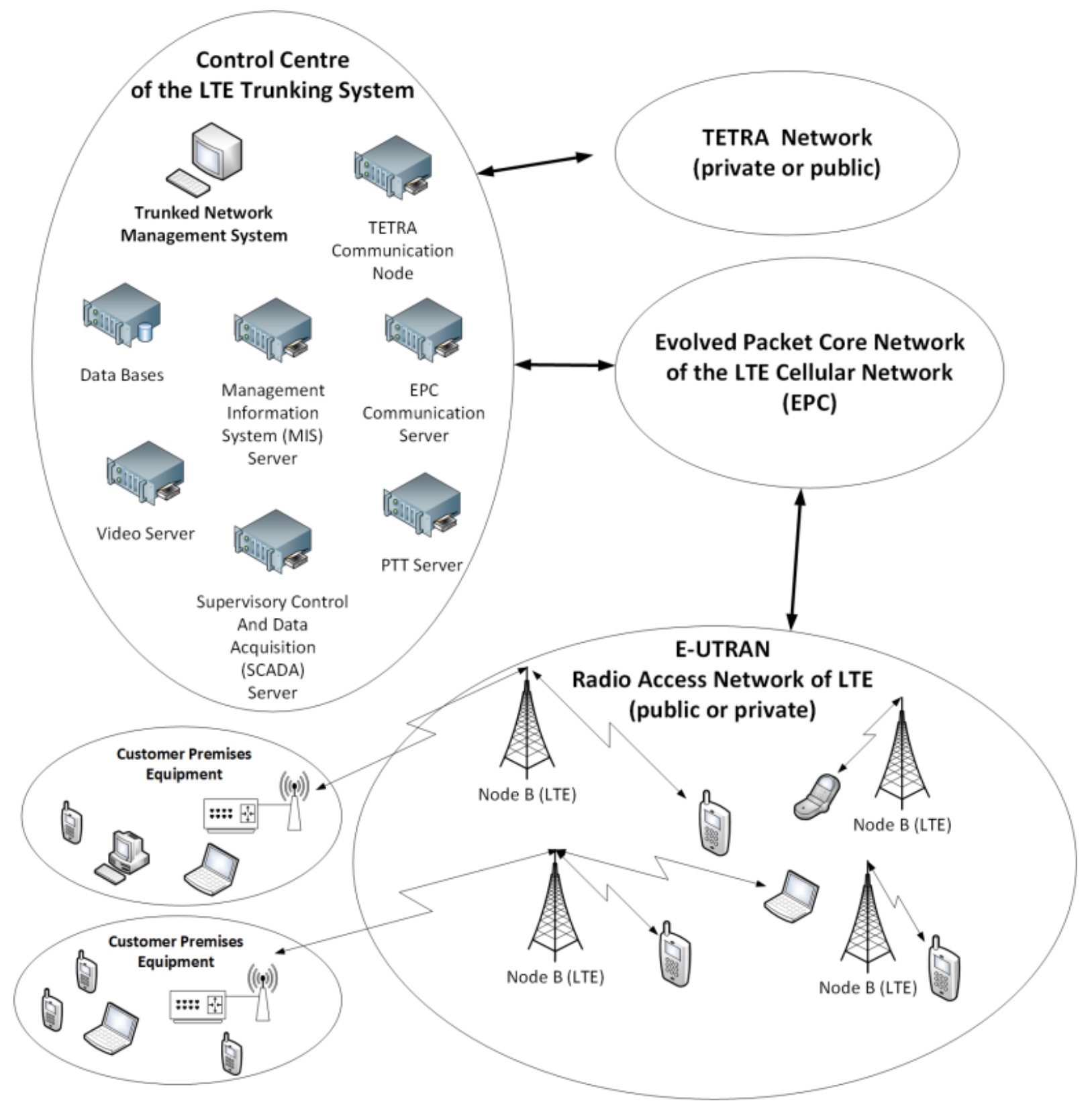

Fig. 1. General concept of the LTE trunked radio.

- Public network (PSTN, PBX, PLMN) and valid communication equipment: fixed-line phones, mobile phones, satellite phones, digital phones.

\section{Dispatch LTE Solution BASED On PRIVATE NETWORK}

LTE is the standard of cellular systems of new generation which is the result of its development towards 4G (LTE Advanced), elaborated by 3GPP. The LTE system provides a much higher bit rate achieved in comparison to UMTS, through the use of efficient techniques for signal processing, multi-antenna techniques and broadband MIMO channels, which may be 1.4, 3, 5, 10, 15 and $20 \mathrm{MHz}$, and for LTEAdvanced up to $100 \mathrm{MHz}$ (5 channels of $20 \mathrm{MHz}$ ) [1].

It provides flexible bandwidth allocation for users and achieve very high transmission rates. The OFDMA
(Orthogonal Frequency Division Multiple Access) is applied in downlink for FDD mode, and the SC-FDMA technique of for uplink (Single Carrier - Frequency Division Multiple Access), also in the FDD mode. But in TDD mode the OFDMA technique is used for both transmission directions.

LTE may ensure the achievement of downlink bit rate even more than $150 \mathrm{Mbps}$ (using 2x2 MIMO technology), and in uplink even more than 80 Mbps [2]. Finally achieved rate depends on the bandwidth and the implementation details of individual operators and equipment suppliers. In the OFDM technique, signal transmission is made using a number of orthogonal subcarriers, which are independently modulated by the different signal streams. These subcarriers are spaced by $15 \mathrm{kHz}$, which means that such $10 \mathrm{MHz}$ band can fit hundreds of mutually orthogonal subcarriers. The diversity of bit rate is achieved by the selection of modulation (e.g. QPSK, 16 QAM, 
and 64 QAM), a coding rate and free allocation of subcarriers for each user (OFDMA).

Trunking concepts of LTE in general rely on the separation of infrastructure dedicated for the purpose of dispatching, both hardware and software, in order to extend the functionality of the LTE system. Additionally, it can be executed regardless of the participation of a mobile operator.

In addition, using a virtual private network tunnels (so called: Virtual Private Network - VPN) achieves a certain isolation from access by unauthorized users and ensure a significant level of transmission security. Such a system can have its own management subsystem and a set of devices and servers supporting its dispatch functions.

Fig. 1 shows an example of the architecture of LTE dispatching system, on the background of general, simplified architecture of LTE mobile public system. As we can see, the basis of the proposed solution is to separate the functional area into an imaginary name Control Centre of the LTE Trunking System, which is the central area of the system. Trunked LTE at this level can communicate and interact with TETRA or DMR to a limited extent, which depends on the adopted solutions dedicated to the institution, service etc.

However, the basis for communication is LTE, what means the connection of dispatching system with architecture of the LTE core network called EPC (Evolved Packet Core network). It is worth to note that the trunking system is a packet switched system, like the LTE cellular system, which gives the possibility to increase the transmission efficiency in comparison with the circuit-switched channels. This solution allows creating internal private VPN network to enhance the security of transmission. In contrast, the TETRA communication system may also take place in the packet mode channels.

Radio access to user terminals is via the LTE radio access network called E-UTRAN (Enhanced - Universal Terrestrial Radio Access Network). It is made using base stations (Node $B)$. In addition, using multi-system terminals the communication can also be made by TETRA.

The area of operation of the end-users is any equipment belongs to the user equipment CPE (Customer Premises Equipment). Then, is possible the creation of internal networks based on additional user equipment and application of additional routing (e.g. Wi-Fi). Of course, the function of user terminals can provide various kinds of devices, including: laptops, smartphones et al. And for CPE we can use telephones, routers, switches, local gateways, set-top-boxes, peripheral devices including mobile terminal equipment, network adapters for local area networks, access points, etc.

\section{MANAgement CentRe of LTE TRUnKed RAdio}

The control centre may, in principle, include a variety of network elements for custom data services and voice services, depending on actual needs and used systems. For instance, it may cooperate with LTE only or provide support to the TETRA system.

The control centre may include:

- Trunked Network Management System,

- Supporting databases,
- EPC Communication Server - communication server to work with the LTE cellular network

- TETRA Communication Node

- Servers for service of Management Information System for companies

- Server for SCADA services (Supervisory Control and Data Acquisition),

- Video server for video services

- Server for PTT services (Push-to-Talk), typical for dispatching systems and others.

The above list is not closed and the actual selection of services and equipment depends on the needs of system customers. As we can see, a significant portion of the listed above devices includes services requiring a wide bandwidth and high transmission rate, which now may be available only in cellular systems, such as LTE. The system can enable the implementation of a number of services, which, in general, were not previously available to the dispatching systems.

These services include, for instance:

- Services of industrial monitoring and data acquisition, socalled SCADA, which may also include broadband transmission of video signals and high-rate data transmission, and facilitate the acquisition of information of all kinds, industrial facilities and military bases, police, etc.,

- Local and extensive management information systems (MIS) - advanced computer systems for business and other organizations, for the recording and analysis of data from various organizational units and their delivery to the superior units (managers), as ordered, current and properly processed form, e.g. storage state, statistics, financial reports etc.

- Video services, including wireless video, e.g. for video industry monitoring of: refineries, gas pipelines, ports, shipyards and others, and for uploading videos of different origin and destination.

\section{TETRA AND LTE INTEGRATION}

Dissemination of LTE dispatching solutions will probably be bigger because the system in terms of achieved data rate is generally considered a breakthrough. So, regardless of the development of TETRA and DMR as dedicated systems for special applications, it is expected both independently operating LTE dispatching solutions and solutions that integrate LTE with TETRA.

It is evident that high-rate data transmission in TETRA will never be possible to the extent as in LTE and never reach such a large degree of modernity. The speed of development of LTE is unrivalled because the potential of companies implementing the LTE is globally much greater than the potential of companies implementing TETRA.

Many companies, however, suggests solutions that integrate TETRA with LTE, what becomes very interesting in the context of LTE trunked radio concepts development. Already some giants in the market of TETRA, which also has its share in LTE design, test devices that can simultaneously handle 


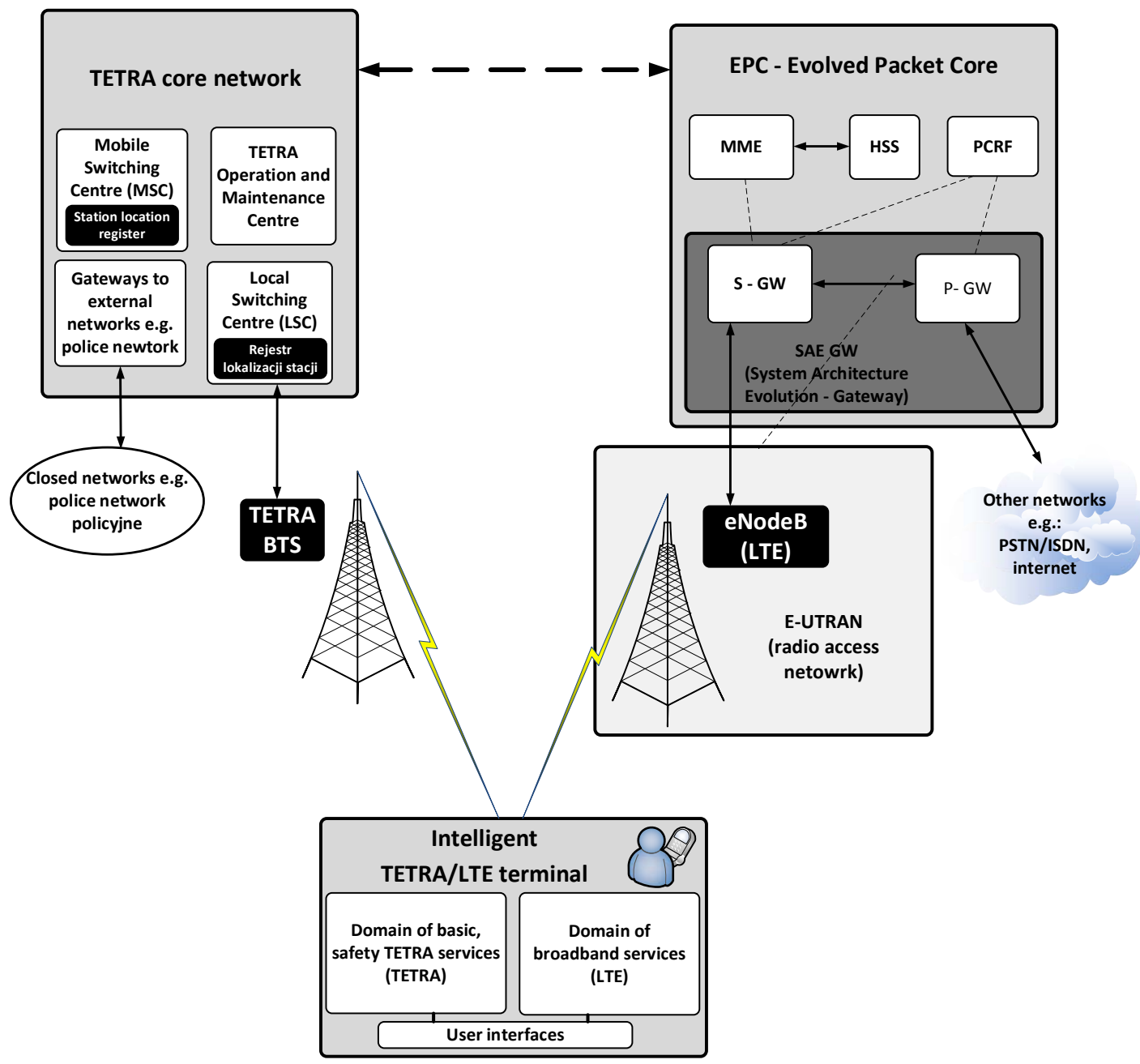

Markers: MME - Mobility Management Entity, HSS - Home Subscribtion Server, P-GW - Packet Data Network Gateway, S-GW - Serving Gateway, eNodeB, PCRF - Policy and Charging Resource Function (module for service management and QoS management)

Fig. 2. Architecture of the system integrating TETRA and LTE, maintaining their infrastructure independence.

both TETRA and LTE. With this approach, TETRA will evolve towards integration with LTE.

One of the concepts of integration process development is to offer the implementation of services "TETRA via LTE" (TETRA communications over LTE) [4]. This proposal includes the launch in LTE the full set of services typical for TETRA and deployment appropriately designed dual-system or multi-system terminals. Such terminals will be mechanically immunized and resistant to exposure, as in classical TETRA.

The original path of TETRA evolution towards LTE includes high-rate data services requiring wide bandwidth, so e.g.: multimedia services, complex database support, image transmission, audiovisual signals transmission etc. In contrast, the core of voice service may long remain rather on the side of TETRA.

The basis of cooperation between TETRA and LTE is the behaviour of the boundary between the terminal software that supports TETRA and LTE support software. This solution is proposed in view of the high level of security of TETRA, which may not always be used. However, thanks to this we are able to provide LTE services while preserving the integrity, containment and high level of safety TETRA, by isolating these two systems.

This problem is analyzed due to the reported needs of services that still avoid the use of open networks. Sure, that with such terms TETRA can continue to remain closed for services and must not be exposed to possible unauthorized access to the public network.

Creating a closed network is a typical method of securing special computer systems from tampering with the outside world.

Fig. 2 shows the system architecture for integrating TETRA with LTE while maintaining their independence. As we can see, there are completely separated areas of network management. The dotted arrow represents only optional network connecting, if any possibility of communication in both networks without user terminals can be desirable. In 
general, however, core networks of these two systems are separated, similar to radio access networks. In the illustrated embodiment, there is no possibility of communication from the TETRA network to external networks supported by LTE and vice versa.

This means that the area of TETRA operation is only possible access to the closed computer network. By contrast, the access to the public network is possible from the user terminal via the LTE network only. In the LTE network may be implemented both voice and broadband services.

Intelligent TETRA-LTE terminal has two completely separable domain, in which are defined only the ability to independently implement basic services TETRA or the possibility of implementing basic services and broadband via LTE network. Thus, it is not possible to reach data sent over the TETRA network with LTE network and vice versa. The whole is functionally connected only by user interfaces.

\section{CONCLUSION}

Presented an overview of solutions and appropriate directions for the development of trunked systems clearly shows that the increasingly important solution is the integration of TETRA with public cellular systems. With such solutions will be possible to maintain high standards of TETRA safety, while ensuring access to public networks and broadband services, which will never be possible to implement in the same TETRA. Particularly noteworthy is the integration of TETRA with LTE.
The development of these solutions will certainly be very dynamic in the coming years. It should be emphasized that LTE may later take over all the functions of the TETRA trunking system for security, enriching it with broadband services, without the use of TETRA.

This relates to the LTE system architecture, speed of access to services, which is constantly optimized, and the structure of its communication interfaces as well as the overall philosophy of system work. If for some time LTE will cover the whole countries, then the use of LTE by services will become more real. Then TETRA can be almost completely absorbed by LTE. At present, however, the more realistic is the use of secure hybrid solutions, which are presented in this paper.

\section{REFERENCES}

[1] M. Brewka, M. Gajewska, S. Gajewski, L. Staszkiewicz, TETRA as sensor system for monitoring and reporting of remote equipment work. In proc. of XII Conference on Automation and Exploitation of Control and Communication Systems (in Polish), ASMOR 2011, Zeszyty Naukowe AMW, nr 185A. Gdynia 2011.

[2] S. Gajewski, Capacity Estimation of the OFDMA and SC-FDMA Radio Interface for UMTS-LTE, Polish Journal of Environmental Studies, Vol. 18, No. 5A. Hard Olsztyn 2009.

[3] S. Gajewski, M. Sokól, M. Gajewska, Data Protection and Crypto Algorithms' Performance in RSMAD, IEEE 73rd Vehicular Technology Conference, VTC Spring 2011, Budapest, Hungary, May 2011.

[4] D. Hartman, M. Stephan, X. Cao, D. Wermser, M. Zeuschner, R. Hunger, F. Andjelo, Initial Development of a SIP-/RTP-based Core Network for the TETRA Mobile Radio System Aiming at Transparent Availability of its Features in LTE, 16 VDE/ITG Fachtagung Mobilkommunikation, Osnabruck 2011. 